\title{
A Syntactic Approach to Combining Functional Notation, Lazy Evaluation, and Higher-Order in LP Systems
}

\author{
Amadeo Casas ${ }^{1}$, Daniel Cabeza ${ }^{2}$, and Manuel V. Hermenegildo ${ }^{1,2}$ \\ 1 Depts. of Comp. Science and Electr. and Comp. Eng., Univ. of New Mexico, USA \\ \{amadeo, herme\}ocs.unm.edu \\ ${ }^{2}$ School of Computer Science, T.U. Madrid (UPM), Spain \\ \{dcabeza, herme\}@fi.upm.es
}

\begin{abstract}
Nondeterminism and partially instantiated data structures give logic programming expressive power beyond that of functional programming. However, functional programming often provides convenient syntactic features, such as having a designated implicit output argument, which allow function call nesting and sometimes results in more compact code. Functional programming also sometimes allows a more direct encoding of lazy evaluation, with its ability to deal with infinite data structures. We present a syntactic functional extension, used in the Ciao system, which can be implemented in ISO-standard Prolog systems and covers function application, predefined evaluable functors, functional definitions, quoting, and lazy evaluation. The extension is also composable with higher-order features and can be combined with other extensions to ISO-Prolog such as constraints. We also highlight the features of the Ciao system which help implementation and present some data on the overhead of using lazy evaluation with respect to eager evaluation.
\end{abstract}

Keywords: Declarative Languages; Logic, Functional, and LogicFunctional Programming; Lazy Evaluation; Higher Order.

\section{Introduction}

Logic Programming offers a number of features, such as nondeterminism and partially instantiated data structures, that give it expressive power beyond that of functional programming. However, certain aspects of functional programming provide in turn syntactic convenience. This includes for example having a syntactically designated output argument, which allows the usual form of function call nesting and sometimes results in more compact code. Also, lazy evaluation, 
which brings the ability to deal with infinite (non-recursive) data structures $[1,2]$, while subsumed operationally by logic programming features such as delay declarations, enjoys a more direct encoding in functional programming. Bringing this syntactic convenience to logic programming can result in a more compact program representation in certain cases and is therefore a desirable objective.

With this objective in mind, in this paper we present a design for an extensive functional syntactic layer for logic programing. While the idea of adding functional features to logic programming systems is clearly not new, and there are currently a good number of systems which integrate functions and higher-order programming into some form of logic programming, we feel that our proposal and its implementation offer a combination of features which make it interesting in itself (see Section 6 for a discussion of related work).

Our approach was inspired by some of the language extension capabilities of the Ciao system [3]: Ciao offers a complete ISO-Prolog system, but one of its most remarkable features is that, through a novel modular design [5], all ISO-Prolog features are library-based extensions to a simple declarative kernel. This allows on one hand not loading any (for example, impure) features from ISO-Prolog when not needed, and on the other hand adding many additional features at the source (Prolog) level, without modifying the compiler or the low-level machinery. The facilities that allow this (grouped under the Ciao packages concept [5]) are the same ones used for implementing the functional extensions proposed herein, and are also the mechanism by which other syntactic and semantic extensions are supported in the system. The latter include constraints, objects, feature terms/records, persistence, several control rules, etc., giving Ciao its multi-paradigm flavor.

However, while the Ciao extension mechanisms make implementation smoother and more orthogonal in our view, ${ }^{1}$ a fundamental design objective and feature of our functional extensions is that they are to a very large extent directly applicable to (and also relatively straightforward to implement in) any modern (ISO-)Prolog system [6], and we hope to contribute in that way to their adoption in such systems. Thus, we will also discuss ISO-Prolog when describing the implementation of the proposed extensions.

The rest of the paper is organized as follows: first, we discuss in Section 2 our general approach to integrating functional notation. Section 3 presents how we implemented this approach in Ciao. Section 4 shows an example of the use of lazy evaluation, and how it is achieved by our implementation. Section 5 presents some experimental results. Finally, section 6 presents our conclusions and discusses related work.

\section{Functional Notation in Ciao}

Basic Concepts and Notation: Our notion of functional notation for logic programming departs in a number of ways from previous proposals. The fundamental one is that functional notation in principle simply provides syntactic

\footnotetext{
${ }^{1}$ As we will try to highlight with the upfront intention of motivating the adoption of
} the extension model by other logic programming systems. 
sugar for defining and using predicates as if they were functions, but they can still retain the power of predicates. In this model, any function definition is in fact defining a predicate, and any predicate can be used as a function. The predicate associated with a function has the same name and one more argument, meant as the place holder for the result of the function. This argument is by default added to the right, i.e., it is the last argument, but this can be changed by using a declaration. The syntax extensions provided for functional notation are the following:

Function applications: Any term preceded by the $\sim$ operator is a function application, as can be seen in the goal write $(\sim \arg (1, T))$, which is strictly equivalent to the sequence $\arg (1, T, A), \operatorname{write}(A)$. To use a predicate argument other than the last as the return argument, a declaration like:

:- fun_return functor $(\sim,-,-)$.

can be used, so that $\sim_{\text {functor }}(f, 2)$ is evaluated to $f\left({ }_{-},-\right)$(where functor $/ 3$ is the standard ISO-Prolog builtin). This definition of the return argument can also be done on the fly in each invocation in the following way: functor $(\sim, f, 2)$. Functors can be declared as evaluable (i.e., being in calls in functional syntax) by using the declaration $f u_{-}$eval/1. This allows avoiding the need to use the operator. Thus, ":- fun_eval arg/2." allows writing write $(\arg (1, T))$ instead of write $(\sim \arg (1, T))$ as above. This declaration can also be used to change the default output argument:

:- fun_eval functor $\left(\sim,{ }_{-},{ }_{-}\right)$.

Note that all these declarations, as is customary in Ciao, are local to the module where they are included.

Predefined evaluable functors: In addition to functors declared with the declaration fun_eval/1, several functors are evaluable, those being:

- The functors used for disjunctive and conditional expressions, (1)/2 and (?) $/ 2$. A disjunctive expression has the form (V1|V2), and its value when first evaluated is V1, and on backtracking V2. A conditional expression has the form (Cond ? V1), or, more commonly, (Cond ? V1 | V2). If the execution of Cond as a goal succeeds the return value is V1. Otherwise in the first form it causes backtracking, and in the second form its value is V2. Due to operator precedences, a nested expression

(Cond1 ? V1 | Cond2 ? V2 | V3)

is evaluated as (Cond1 ? V1 | (Cond2 ? V2 | V3)).

- If the declaration :- fun_eval arith(true) is used, all the functors understood by is $/ 2$ are considered evaluable (they will be translated to a call to is $/ 2$ ). This is not active by default because several of those functors, like $(-) / 2$ or $(/) / 2$, are traditionally used in Prolog for creating structures. Using false instead of true the declaration can be disabled.

Functional definitions: A functional definition is composed of one or more functional clauses. A functional clause is written using the binary operator $:=$, as in opposite(red) := green.

Functional clauses can also have a body, which is executed before the result value is computed. It can serve as a guard for the clause or to provide the equivalent of where-clauses in functional languages: 
fact $(0):=1$.

$\operatorname{fact}(\mathrm{N}):=\mathrm{N} * \sim \operatorname{fact}(--N):-N>0$.

Note that guards can often be defined more compactly using conditional expressions:

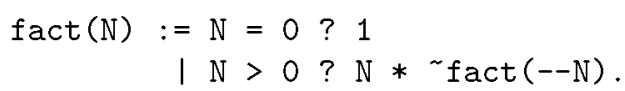

If the declaration :- fun_eval defined(true) is active, the function defined in a functional clause does not need to be preceded by (for example the $\mathrm{fact}(-\mathrm{N})$ calls above).

The translation of functional clauses has the following properties:

- The translation produces steadfast predicates [7], that is, output arguments are unified after possible cuts.

- Defining recursive predicates in functional style maintains the tail recursion of the original predicate, thus allowing the usual compiler optimizations.

Quoting functors: Functors (either in functional or predicate clauses) can be prevented from being evaluated by using the $\left(^{n}\right) / 1$ prefix operator (read as "quote"), as in:

$\operatorname{pair}(A, B):={ }^{-}(A-B)$.

Note that this just prevents the evaluation of the principal functor of the enclosed term, not the possible occurrences of other evaluable functors inside.

Scoping: When using function applications inside the goal arguments of metapredicates, there is an ambiguity as they could be evaluated either in the scope of the outer execution or in the scope of the inner execution. The default behavior is to evaluate function applications in the scope of the outer execution. If they should be evaluated in the inner scope the goal containing the function application needs to be escaped with the $\left(^{(n)}\right) / 1$ prefix operator, as in $\mathrm{findall}\left(\mathrm{X},\left(\mathrm{d}(\mathrm{Y}),{ }^{\cdots}\left(\mathrm{X}={ }^{\sim} \mathrm{f}(\mathrm{Y})+1\right)\right), \mathrm{L}\right)$ (which could also be written as $f$ indall $(X, \cdots(d(Y), X=\sim f(Y)+1), L))$, and whose expansion is $\mathrm{findall}(\mathrm{X},(\mathrm{d}(\mathrm{Y}), \mathrm{f}(\mathrm{Y}, \mathrm{Z}), \mathrm{T}$ is $\mathrm{Z}+1, \mathrm{X}=\mathrm{T}), \mathrm{L})$. With no escaping the function application is evaluated in the scope of the outer execution, i.e., $f(Y, Z), T$ is $Z+1$, findall $(X,(d(Y), X=T), L)$.

Laziness: Lazy evaluation is a program evaluation technique used particularly in functional languages. When using lazy evaluation, an expression is not evaluated as soon as it is assigned, but rather when the evaluator is forced to produce the value of the expression. The when, freeze, or block control primitives present in many modern logic programming systems are more powerful operationally than lazy evaluation. However, they lack the simplicity of use and cleaner semantics of functional lazy evaluation. In our design, a function (or predicate) can be declared as lazy via the declarations:

:- lazy fun_eval function_name/N.

(or, equivalently in predicate version, ": - lazy pred_name/M.", where $M=$ $N+1$ ). In order to achieve the intended behavior, the execution of each function declared as lazy is suspended until the return value of the function 


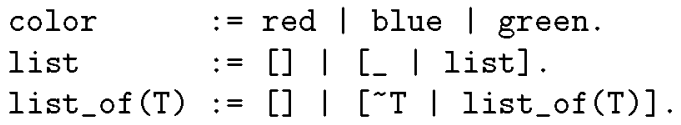

which are equivalent to (note the use of higher-order in the third example):

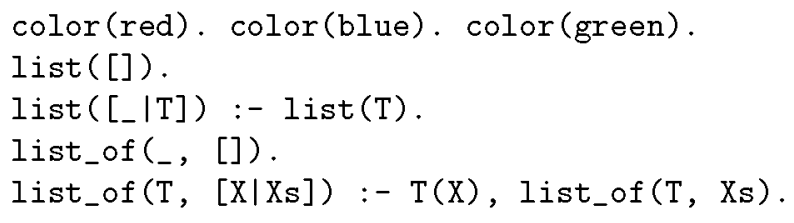

Such types and properties are then admissible in Ciao-style assertions [8], such as the following, and which can be added to the corresponding definitions and checked by the preprocessor or turned into run-time tests [9]:

$:-$ pred append $/ 3::$ list * list * list.

:- pred color_value/2 : : list(color) * int.

Example 3. The combination of functional syntax and user-defined operators brings significant flexibility, as can be seen in the following definition of a list concatenation (append) operator: ${ }^{2}$

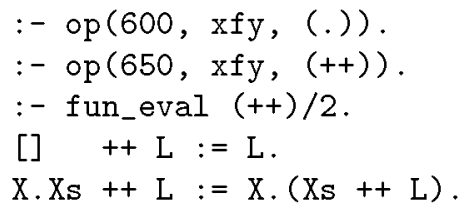

This definition will be compiled exactly to the standard definition of append in Prolog (and, thus, will be reversible). The functional syntax and user-defined operators allow writing for example write ("Hello" ++ Spc ++ "world!") instead of the equivalent forms write( append("Hello", append (Spc, "world!"))) (if append/2 is defined as evaluable) or append(Spc, "world!", T1), append("Hello", T1, T2), write(T2).

Example 4. As another example, we define an array indexing operator for multidimensional arrays. Assume that arrays are built using nested structures whose main functor is 'a' and whose arities are determined by the specified dimensions, i.e., a two-dimensional array $A$ of dimensions $[N, M]$ will be represented by the nested structure a $\left(\mathrm{a}\left(A_{11}, \ldots, A_{1 M}\right), \mathrm{a}\left(A_{21}, \ldots, A_{2 M}\right), \ldots, \mathrm{a}\left(A_{N 1}, \ldots\right.\right.$, $\left.A_{N M}\right)$ ), where $A_{11}, \ldots A_{N M}$ may be arbitrary terms. ${ }^{3}$ The following recursive definition defines the property array/2 and also the array access operator (a:

\footnotetext{
${ }^{2}$ This operator, as well of other conveniences to be able to program in a more functional-flavored style, are defined in an additional Ciao package.

${ }^{3}$ We ignore for simplicity possible arity limitations, solved in any case typically by further nesting with logarithmic access time (as in Warren/Pereira's classical library).
} 


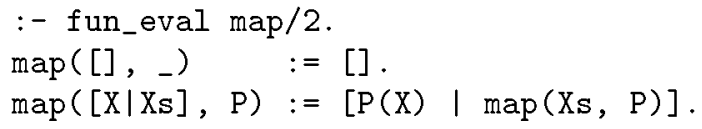

With this definition, after calling:

["helloworld", "byeworld"] = map (["hello", "bye"], ++(X)).

(where $(++) / 2$ corresponds to the above definition of append) $\mathrm{X}$ will be bound to "world", which is the only solution to the equation. Also, when calling:

$\operatorname{map}(L,++(X)$, ["hello.", "bye."]).

several values for $\mathrm{L}$ and $\mathrm{X}$ are returned through backtracking:

$\mathrm{L}=$ ["hello", "bye"], $\mathrm{X}=$ ". " ? ;

$\mathrm{L}=[$ "hello.","bye."], X = [] ?

\section{Implementation Details}

As mentioned previously, certain Ciao features have simplified the proposed extension to handle functional notation. In the following we introduce the features of Ciao that were used and how they were applied in this particular application.

Code Translations in Ciao. Traditionally, Prolog systems have included the possibility of changing the syntax of the source code through the use of the op/3 builtin/directive. Furthermore, in many Prolog systems it is also possible to define expansions of the source code (essentially, a very rich form of "macros") by allowing the user to define (or extend) a predicate typically called term_expansion/2 [13,14]. This is usually how, e.g., definite clause grammars (DCG's) are implemented.

However, these features, in their original form, pose many problems for modular compilation or even for creating sensible standalone executables. First, the definitions of the operators and, specially, expansions are often global, affecting a number of files. Furthermore, it is not possible to determine statically which files are affected, because these features are implemented as a side-effect, rather than a declaration: they become active immediately after being read by the code processor (top-level, compiler, etc.) and remain active from then on. As a result, it is impossible just by looking at a source code file to know if it will be affected by expansions or definitions of operators, which may completely change what the compiler really sees, since those may be activated by the load of other, possibly unrelated, files.

In order to solve these problems, the syntactic extension facilities were redesigned in Ciao, so that it is still possible to define source translations and operators, but such translations are local to the module or user file defining them [5]. Also, these features are implemented in a way that has a well-defined behavior in the context of a standalone compiler, separate compilation, and global analysis (and this behavior is implemented in the Ciao compiler, ciaoc [15]). In particular, the load_compilation_module/1 directive allows separating code that will be used at compilation time (e.g., the code used for program 
transformations) from code which will be used at run-time. It loads the module defined by its argument into the compiler.

In addition, in order to make the task of writing source translations easier, the effects usually achieved through term_expansion/ 2 can be obtained in Ciao by means of four different, more specialized directives, which, again, affect only the current module and are (by default) only active at compile-time. The proposed functional syntax is implemented in Ciao using these source translations. In particular, we have used the add_sentence_trans/1 and add_goal_trans/1 directives. A sentence translation is a predicate which will be called by the compiler to possibly convert each term (clause, fact, directive, input, etc.) read by the compiler to a new term, which will be used in place of the original term. A goal translation is a predicate which will be called by the compiler to possibly convert each goal present in each clause of the current text to another goal which replaces the original one. The proposed model can be implemented in Prolog systems similarly using the traditional term_expansion/2 and operator declarations, but having operators and syntactic transformation predicates local to modules is the key to making the approach scalable and amenable to combination with other packages and syntactic extensions in the same application.

Ciao Packages. Packages in Ciao are libraries which define extensions to the language, and have a well defined and repetitive structure. These libraries typically consist of a main source file which defines only some declarations (operator declarations, declarations loading other modules into the compiler or the module using the extension, etc.). This file is meant to be included as part of the file using the library, since, because of their local effect, such directives must be part of the code of the module which uses the library. Any auxiliary code needed at compile-time (e.g., translations) is included in a separate module which is to be loaded into the compiler via a load_compilation_module/1 directive placed in the main file. Also, any auxiliary code to be used at run-time is placed in another module, and the corresponding use_module declaration is also placed in the include file.

In our implementation of functional notation in Ciao we have provided two packages: one for the bare function features without lazy evaluation, and an additional one to provide the lazy evaluation features. The reason for this is that in many cases the lazy evaluation features are not needed and thus the translation procedure is simplified.

The Ciao Implementation of Functional Extensions. Totranslate the functional definitions, we have used as mentioned above the add_sentence_trans/ 1 directive to provide a translation procedure which transforms each functional clause to a predicate clause, adding to the function head the output argument, in order to convert it to the predicate head. This translation procedure also deals with functional applications in heads, as well as with fun_eval directives. Furthermore, all function applications are translated to an internal normal form.

On the other hand, we have used the add_goal_trans/1 directive to provide a translation procedure for dealing with function applications in bodies (which were previously translated to a normal form). The rationale for using a goal 
translation is that each function application inside a goal will be replaced by a variable, and the goal will be preceded by a call to the predicate which implements the function in order to provide a value for that variable. A simple recursive application of this rule achieves the desired effect.

An additional sentence translation is provided to handle the lazy directives. The translation of a lazy function into a predicate is done in two steps. First, the function is converted into a predicate using the procedure sketched above. Then, the resulting predicate is transformed in order to suspend its execution until the value of the output variable is needed. We explain the transformation in terms of the freeze/ 1 control primitive that many modern logic programming systems implement quite efficiently [16], since it is the most widespread (but obviously when $[17]$ or, specially, the more efficient block [16] declarations can also be used). This transformation renames the original predicate to an internal name and add a bridge predicate with the original name which invokes the internal predicate through a call to freeze/2, with the last argument (the output of the function) as suspension variable. This will delay the execution of the internal predicate until its result is required, which will be detected as a binding (i.e., demand) of its output variable. The following section will provide a detailed example of the translation of a lazy function. The implementation with block is even simpler since no bridge predicate is needed.

We show below, for reference, the main files for the Ciao library packages f syntax:

$\%$ fsyntax.pl

:- include(library('fsyntax/ops')). \% Operator definitions

:- load_compilation_module(library ('fsyntax/functionstr')) .

:- add_sentence_trans (defunc/3).

:- add_goal_trans(defunc_goal/3).

and lazy (which will usually be used in conjunction with the first one):

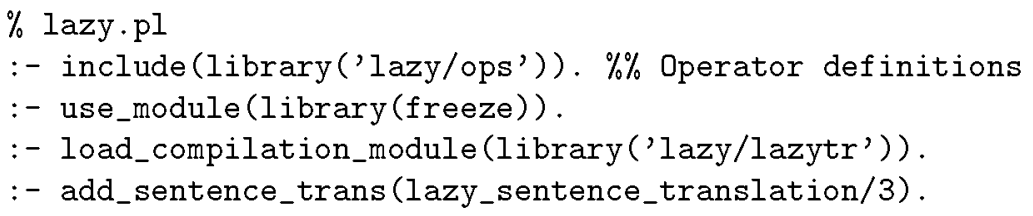

These files will be included in any file that uses the package. The Ciao system source provides the actual detailed code, which follows the our description.

\section{Lazy Functions: An Example}

In this section we show an example of the use of lazy evaluation, and how a lazy function is translated by our Ciao package. Figure 1 shows in the first row the definition of a lazy function which returns the infinite list of Fibonacci numbers, in the second row its translation into a lazy predicate ${ }^{6}$ (by the fsyntax package)

\footnotetext{
${ }^{6}$ The :- lazy fun $-e v a l$ fiblist/0. declaration is converted into a :- lazy fiblist/1. declaration.
} 


\begin{tabular}{|c|}
\hline 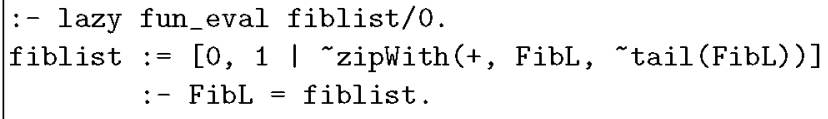 \\
\hline $\begin{array}{l}:- \text { lazy fiblist } / 1 . \\
\text { fiblist([0, } 1 \text { | Rest]) :- } \\
\text { fiblist(FibL) } \\
\text { tail (FibL, T), } \\
\text { zipWith(+, FibL, T, Rest). }\end{array}$ \\
\hline $\begin{array}{l}\text { fiblist(X) }:- \\
\quad \text { freeze }(X, \text { fiblist_lazy_} \$ \$ \$(X)) . \\
\text { fiblist_lazy_\$} \$ \$([0,1 \text { । Rest }]):- \\
\quad \text { fiblist(FibL), } \\
\quad \text { tail(FibL, T) } \\
\quad \text { zipWith(+, FibL, T, Rest). }\end{array}$ \\
\hline
\end{tabular}

Fig. 1. Code translation for a Fibonacci function, to be evaluated lazily

and in the third row the expansion of that predicate to emulate lazy evaluation (where fiblist_lazy $\$ \$$ stands for a fresh predicate name).

In the $\mathrm{fiblist}$ function defined, any element in the resulting infinite list of Fibonacci numbers can be referenced, as, for example, $n t h\left(X,{ }^{\sim}\right.$ fiblist, Value). The other functions used in the definition are tail/2, which is defined as lazy and returns the tail of a list; zipwith/3, which is also defined as lazy and returns a list whose elements are computed by a function having as arguments the successive elements in the lists provided as second and third argument $;^{7}$ and $(+) / 2$ which is defined as by the rule $+(\mathrm{X}, \mathrm{Y}):=\mathrm{Z}:-\mathrm{Z}$ is $\mathrm{X}+\mathrm{Y}$.

Note that the zipWith/3 function (respectively the zipWith/4 predicate) is in fact a higher-order function (resp. predicate).

\section{Some Performance Measurements}

Since the functional extensions proposed simply provide a syntactic bridge between functions and predicates, there are only a limited number of performance issues worth discussing. For the case of real functions, it is well known that performance gains can be obtained from the knowledge that the corresponding predicate is moded (all input arguments are ground and the "designated output" will be ground on output), determinate, non-failing, etc. $[18,20]$. In Ciao this information can in general (i.e., for any predicate or function) be inferred by the Ciao preprocessor or declared with Ciao assertions $[9,8]$. As mentioned

\footnotetext{
${ }^{7}$ It has the same semantics as the zipwith function in Haskell.
} 


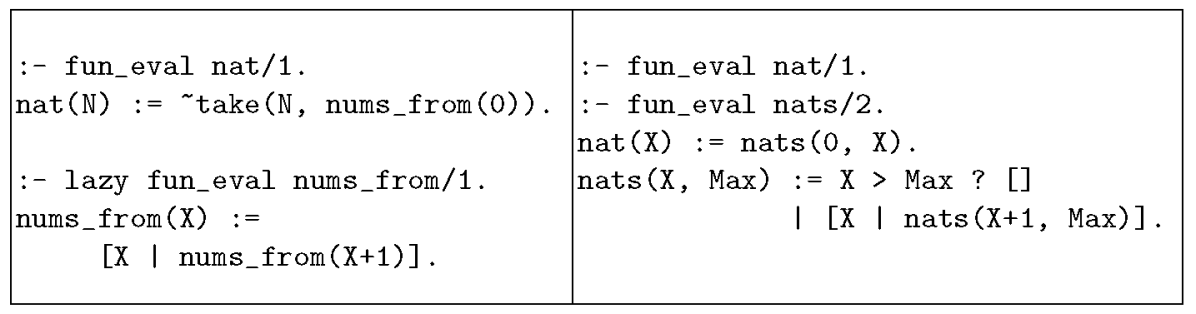

Fig. 2. Lazy and eager versions of function nat $(X)$

before, for declared "real" (func) functions, the corresponding information is added automatically. Some (preliminary) results on current Ciao performance when this information is available are presented in [20].

In the case of lazy evaluation of functions, the main goal of the technique presented herein is not really any increase in performance, but achieving new functionality and convenience through the use of code translations and delay declarations. However, while there have also been some studies of the overhead introduced by delay declarations and their optimization (see, e.g., [21]), it is interesting to see how this overhead affects our implementation of lazy evaluation by observing its performance. Consider the nat/ 2 function in Figure 2, a simple function which returns a list with the first $N$ numbers from an (infinite) list of natural numbers.

Function take/2 in turn returns the list of the first $N$ elements in the input list. This nat $(N)$ function cannot be directly executed eagerly due to the infinite list provided by the nums_from(X) function, so that, in order to compare time and memory results between lazy and eager evaluation, an equivalent version of that function is provided.

Table 1 reflects the time and memory overhead of the lazy evaluation version of nat $(X)$ and that of the equivalent version executed eagerly. As a further example, Table 2 shows the results for a quicksort function executed lazily in comparison to the eager version of this algorithm. All the results were obtained by averaging ten runs on a medium-loaded Pentium IV Xeon 2.0Ghz, 4Gb of RAM memory, running Fedora Core 2.0, with the simple translation of Figure 1, and compiled to traditional bytecode (no global optimizations or native code).

We can observe in both tables that there is certainly an impact on the execution time when functions are evaluated lazily, but even with this version the results are quite acceptable if we take into account that the execution of the predicate does really suspend. Related to memory consumption we show heap sizes, without garbage collection (in order to observe the raw memory consumption rate). Lazy evaluation implies as expected some memory overhead due to the need to copy (freeze) program goals into the heap. Also, while comparing with standard lazy functional programming implementations is beyond the scope of this paper, some simple tests done for sanity check purposes (with HUGS) show that the results are comparable, our implementation being for example slower on nat but faster on qsort, presumably due to the different optimizations being performed by the compilers. 
Table 1. Performance for nat/2 (time in ms. and heap sizes in bytes)

\begin{tabular}{|l||r|r||r|r|}
\cline { 2 - 5 } \multicolumn{1}{c|}{} & \multicolumn{2}{c|}{ Lazy Evaluation } & \multicolumn{2}{c|}{ Eager Evaluation } \\
\hline List & Time & Heap & Time & Heap \\
\hline \hline 10 elements & 0.030 & 1503.2 & 0.002 & 491.2 \\
\hline 100 elements & 0.276 & 10863.2 & 0.016 & 1211.2 \\
\hline 1000 elements & 3.584 & 104463.0 & 0.149 & 8411.2 \\
\hline 2000 elements & 6.105 & 208463.2 & 0.297 & 16411.2 \\
\hline 5000 elements & 17.836 & 520463.0 & 0.749 & 40411.2 \\
\hline 10000 elements & 33.698 & 1040463.0 & 1.277 & 80411.2 \\
\hline
\end{tabular}

Table 2. Performance for qsort/2 (time in ms. and heap sizes in bytes)

\begin{tabular}{|l||r|r||r|r|}
\cline { 2 - 5 } \multicolumn{1}{c|}{} & \multicolumn{2}{c|}{ Lazy Evaluation } & \multicolumn{2}{c|}{ Eager Evaluation } \\
\hline List & Time & Heap & Time & Heap \\
\hline \hline 10 elements & 0.091 & 3680.0 & 0.032 & 1640.0 \\
\hline 100 elements & 0.946 & 37420.0 & 0.322 & 17090.0 \\
\hline 1000 elements & 13.303 & 459420.0 & 5.032 & 253330.0 \\
\hline 5000 elements & 58.369 & 2525990.0 & 31.291 & 1600530.0 \\
\hline 15000 elements & 229.756 & 8273340.0 & 107.193 & 5436780.0 \\
\hline 20000 elements & 311.833 & 11344800.0 & 146.160 & 7395100.0 \\
\hline
\end{tabular}

An example when lazy evaluation can be a better option than eager evaluation in terms of performance (and not only convenience) can be found in a concurrent or distributed system environment (such as, e.g., [22]), and in the case of Ciao also within the active modules framework [3,23]. The example in Figure 3 uses a function, defined in an active module, which returns a big amount of data. Function test/ 0 in module module1 needs to execute function squares/ 1 , in (active, i.e., remote) module module2, which will return a very long list (which could be infinite for our purposes). If squares/1 were executed eagerly then the entire list would be returned, to immediately execute the takewhile/2 function with the entire list. takeWhile/ 2 returns the first elements of a (possibly infinite) list while the specified condition is true. But creating the entire initial list is very wasteful in terms of time and memory requirements. In order to solve this problem, the squares/ 1 function could be moved to module module1 and merged with takewhile/2 (or, also, they could exchange a size parameter). But rearranging the program is not always possible and it may also perhaps complicate other aspects of the overall design.

If on the other hand squares/1 is evaluated lazily, it is possible to keep the definitions unchanged and in different modules, so that there will be a smaller time and memory penalty for generating and storing the intermediate result. As more values are needed by the takewhile/2 function, more values in the list returned by squares/ 1 are built (in this example, only while the new generated value is less than 10000), considerably reducing the time and memory consumption that the eager evaluation would take. 


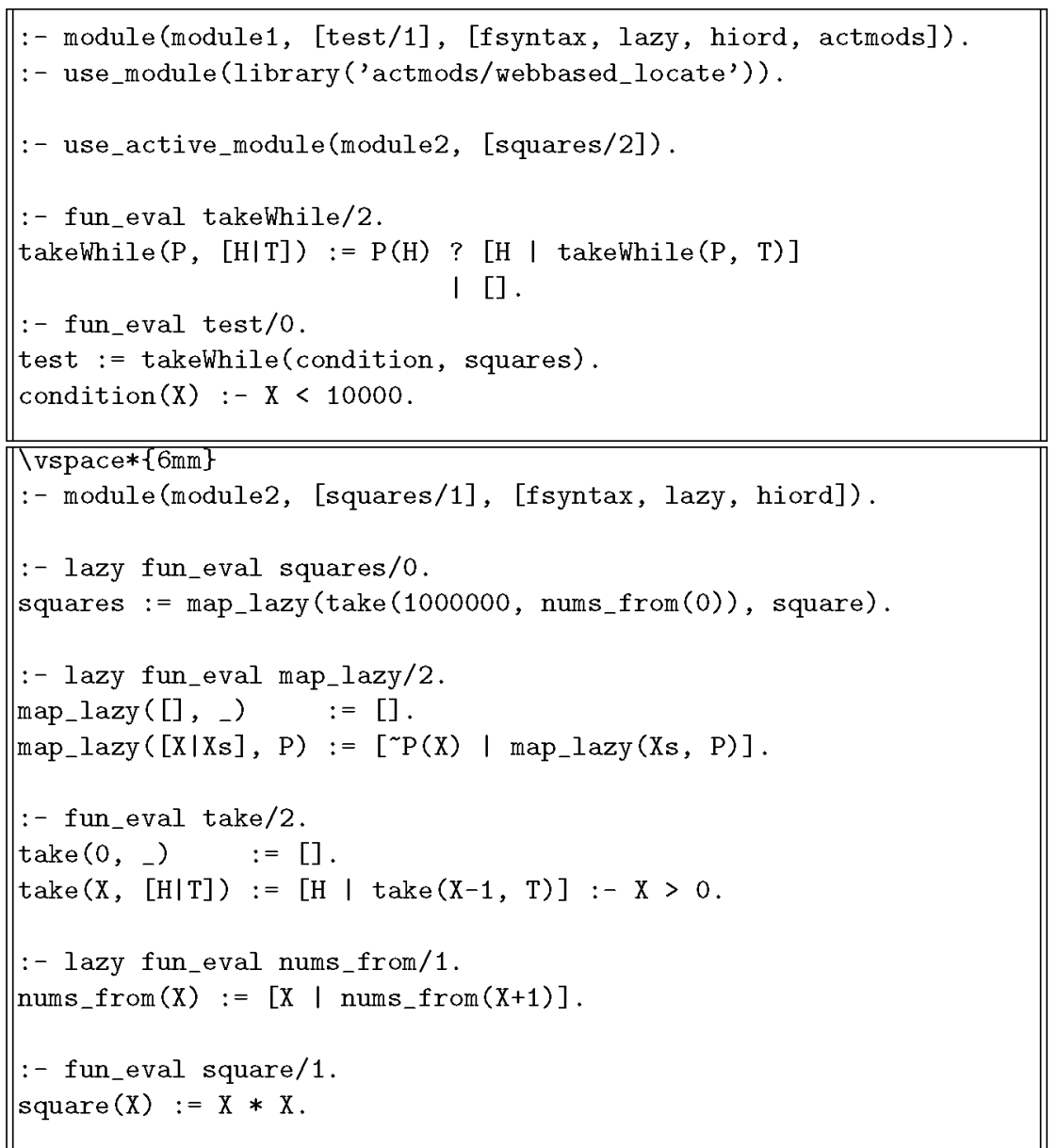

Fig. 3. A distributed (active module) application using lazy evaluation

\section{Conclusions and Related Work}

As mentioned in the introduction, the idea of adding functional features to logic programming systems is clearly not new [24, 25, 17] and there are currently a good number of systems which integrate functions and higher-order programming into some form of logic programming. However, we feel that our proposal and its implementation offer a combination of features which make it interesting in itself. More concretely, the approach is completely syntactic, functions can be limited or retain the power of predicates, any predicate can be called through functional syntax, and lazy evaluation is supported both for functions and predicates. Furthermore, functional syntax can be combined with numerous (Ciao) syntactic and semantic extensions such as higher-order, assertions, records, constraints, objects, persistence, other control rules, etc., without any modification to the compiler or abstract ma- 
chine. Finally, and perhaps most importantly, and again because of the syntactic nature of the extensions, they can be the target of analysis, optimization, static checking, and verification (of types, modes, determinacy, nonfailure, cost, etc.), as performed by, e.g., the Ciao preprocessor [9]. Finally, another important characteristic of our approach is that most of it can be applied directly (or with minor changes) to any ISO-standard Prolog system.

The original version of the functional extensions was first distributed in Ciao 0.2 [4] and later used as an example in [5]. The full description presented herein includes some minor changes with respect to the currently distributed version [3] which will be available in the next release. The performance of the package for lazy evaluation was tested in this system with several examples. As expected, lazy evaluation implies time and memory overhead, which justifies making lazy evaluation optional via a declaration.

Returning to the issue of related work, Lambda Prolog [26] offers a highly expressive language with extensive higher-order programming features and lambdaterm (pattern) unification. On the other hand it pays in performance the price of being "higher order by default," and is not backwards compatible with traditional Prolog systems. It would be clearly interesting to support pattern unification, but we propose to do it as a further (and optional) extension, and some work is in progress along these lines. HiLog [27] is a very interesting logic programming system (extending XSB-Prolog) which allows using higher-order syntax, but it does not address the issue of supporting functional syntax or lazyness. Functional-logic systems such as Curry or Babel [31,32] perform a full integration of functional and logic programming, with higher-order support. On the other hand, their design starts from a lazy functional syntax and semantics, and is strongly typed. However, it may also be interesting to explore supporting narrowing as another optional extension. Mercury [28] offers functional and higher-order extensions based on Prolog-like syntax, but they are an integral part of the language (as opposed to an optional extension) and, because of the need for type and mode declarations, the design is less appropriate for non strongly-typed, unmoded systems. As mentioned above, in our design type and mode declarations are optional and handled separately through the assertion mechanism. Also, Mercury's language design includes a number restrictions with respect to Prolog-like systems which bring a number of implementation simplifications. In particular, the modedness (no unification) of Mercury programs brings them much closer to the functional case. As a result of these restrictions, Mercury always performs the optimizations pointed out when discussing our funct declaration (or when that type of information is inferred by CiaoPP). ${ }^{8}$ $\mathrm{Oz}[30]$ also allows functional and (a restricted form of) logic programming, and supports higher-order in an untyped setting, but its syntax and semantics are quite different from those of LP systems. BIM Prolog offered similar functionality to our $\sim / 2$ operator but, again, by default and as a builtin.

\footnotetext{
${ }^{8}$ However, recent extensions to support constraints [29] recover unification, including the related implementation overheads and mechanisms (such as the trail), and will require analysis for optimization, moving Mercury arguably closer to Ciao in design.
} 


\section{References}

1. Narain, S.: Lazy evaluation in logic programming. In: Proc. 1990 Int. Conference on Computer Languages. (1990) 218-227

2. Antoy, S.: Lazy evaluation in logic. In: Symp. on Progr. Language Impl. and Logic Progr (PLILP'91), Springer Verlag (1991) 371-382 LNCS 528.

3. Bueno, F., Cabeza, D., Carro, M., Hermenegildo, M., López-García, P., (Eds.), G.P.: The Ciao System. Reference Manual (v1.10). The ciao system documentation series-TR, School of Computer Science, Technical University of Madrid (UPM) (2004) System and on-line version of the manual available at http://clip.dia.fi.upm.es/Software/Ciao/.

4. Bueno, F., Cabeza, D., Carro, M., Hermenegildo, M., López-García, P., Puebla, G.: The Ciao Prolog System. Reference Manual. The Ciao System Documentation Series-TR CLIP3/97.1, School of Computer Science, Technical University of Madrid (UPM) (1997) System and on-line version of the manual available at http://clip.dia.fi.upm.es/Software/Ciao/.

5. Cabeza, D., Hermenegildo, M.: A New Module System for Prolog. In: International Conference on Computational Logic, CL2000. Number 1861 in LNAI, SpringerVerlag (2000) 131-148

6. Deransart, P., Ed-Dbali, A., Cervoni, L.: Prolog: The Standard. Springer-Verlag (1996)

7. O'Keefe, R.: The Craft of Prolog. MIT Press (1990)

8. Puebla, G., Bueno, F., Hermenegildo, M.: An Assertion Language for Constraint Logic Programs. In Deransart, P., Hermenegildo, M., Maluszynski, J., eds.: Analysis and Visualization Tools for Constraint Programming. Number 1870 in LNCS. Springer-Verlag (2000) 23-61

9. Hermenegildo, M.V., Puebla, G., Bueno, F., López-García, P.: Integrated Program Debugging, Verification, and Optimization Using Abstract Interpretation (and The Ciao System Preprocessor). Science of Computer Programming 58 (2005) 115-140

10. Cabeza, D., Hermenegildo, M.: Higher-order Logic Programming in Ciao. Technical Report CLIP7/99.0, Facultad de Informática, UPM (1999)

11. Cabeza, D.: An Extensible, Global Analysis Friendly Logic Programming System. PhD thesis, Universidad Politécnica de Madrid (UPM), Facultad Informatica UPM, 28660-Boadilla del Monte, Madrid-Spain (2004)

12. Cabeza, D., Hermenegildo, M., Lipton, J.: Hiord: A Type-Free Higher-Order Logic Programming Language with Predicate Abstraction. In: Ninth Asian Computing Science Conference (ASIAN'04). Number 3321 in LNCS, Springer-Verlag (2004) 93-108

13. Quintus Computer Systems Inc. Mountain View CA 94041: Quintus Prolog User's Guide and Reference Manual-Version 6. (1986)

14. Carlsson, M., Widen, J.: Sicstus Prolog User's Manual, Po Box 1263, S-16313 Spanga, Sweden. (1994)

15. Cabeza, D., Hermenegildo, M.: The Ciao Modular Compiler and Its Generic Program Processing Library. In: ICLP'99 WS on Parallelism and Implementation of (C)LP Systems, N.M. State U. (1999) 147-164

16. Carlsson, M.: Freeze, Indexing, and Other Implementation Issues in the Wam. In: Fourth International Conference on Logic Programming, University of Melbourne, MIT Press (1987) 40-58 
17. Naish, L.: Adding equations to NU-Prolog. In: Proceedings of The Third International Symposium on Programming Language Implementation and Logic Programming (PLILP'91). Number 528 in Lecture Notes in Computer Science, Passau, Germany, Springer-Verlag (1991) 15-26

18. Van Roy, P.: 1983-1993: The Wonder Years of Sequential Prolog Implementation. Journal of Logic Programming 19/20 (1994) 385-441

19. Henderson et al., F.: (The Mercury Language Reference Manual) URL: http:// www.cs.mu.oz.au/research/mercury/information/doc/reference_manual_ toc.html.

20. Morales, J., Carro, M., Hermenegildo, M.: Improving the Compilation of Prolog to C Using Moded Types and Determinism Information. In: Proceedings of the Sixth International Symposium on Practical Aspects of Declarative Languages. Number 3507 in LNCS, Heidelberg, Germany, Springer-Verlag (2004) 86-103

21. Marriott, K., de la Banda, M.G., Hermenegildo, M.: Analyzing Logic Programs with Dynamic Scheduling. In: 20th. Annual ACM Conf. on Principles of Programming Languages, ACM (1994) 240-254

22. Carro, M., Hermenegildo, M.: A simple approach to distributed objects in prolog. In: Colloquium on Implementation of Constraint and LOgic Programming Systems (ICLP associated workshop), Copenhagen (2002)

23. Cabeza, D., Hermenegildo, M.: Distributed Concurrent Constraint Execution in the CIAO System. In: Proc. of the 1995 COMPULOG-NET Workshop on Parallelism and Implementation Technologies, Utrecht, NL, U. Utrecht / T.U. Madrid (1995) Available from http://www.clip.dia.fi.upm.es/.

24. Barbuti, R., Bellia, M., Levi, G., Martelli, M.: On the integration of logic programming and functional programming. In: International Symposium on Logic Programming, Atlantic City, NJ, IEEE Computer Society (1984) 160-168

25. Bellia, M., Levi, G.: The relation between logic and functional languages. Journal of Logic Programming 3 (1986) 217-236

26. Nadathur, G., Miller, D.: An overview of $\lambda$ prolog. In: Proc. 5 th Conference on Logic Programming \& 5th Symposium on Logic Programming (Seattle), MIT Press (1988) 810-827

27. Chen, W., Kifer, M., Warren, D.: HiLog: A foundation for higher order logic programming. Journal of Logic Programming 15 (1993) 187-230

28. Somogyi, Z., Henderson, F., Conway, T.: The execution algorithm of Mercury: an efficient purely declarative logic programming language. JLP 29 (1996)

29. Becket, R., de la Banda, M.G., Marriott, K., Somogyi, Z., Stuckey, P.J., Wallace, M.: Adding constraint solving to mercury. In: Eight International Symposium on Practical Aspects of Declarative Languages. Number 2819 in LNCS, SpringerVerlag (2006) 118-133

30. Haridi, S., Franzén, N.: The Oz Tutorial. DFKI. (2000) Available from http://www.mozart-oz.org.

31. Hanus et al, M.: Curry: An Integrated Functional Logic Language. (http://www. informatik. uni-kiel. de/ ${ }^{\sim m h} /$ curry/report.html)

32. Moreno Navarro, J., Rodríguez-Artalejo, M.: BABEL: A functional and logic programming language based on constructor discipline and narrowing. In: Conf. on Algebraic and Logic Programming (ALP). LNCS 343 (1989) 223-232 